\title{
Automatic 4-D Registration in Dynamic MR Renography Based on Over-Complete Dyadic Wavelet and Fourier Transforms
}

\author{
Ting Song ${ }^{1}$, Vivian S. Lee ${ }^{2}$, Henry Rusinek ${ }^{2}$, Manmeen Kaur ${ }^{2}$, \\ and Andrew F. Laine ${ }^{1}$ \\ ${ }^{1}$ Department of Biomedical Engineering, Columbia University, \\ New York, NY, U.S.A \\ \{ts2060, laine\} @columbia.edu \\ ${ }^{2}$ Department of Radiology, New York University School of Medicine, \\ New York, NY, U.S.A \\ \{Vivian.Lee, manmeen.kaur\}@med.nyu.edu, hr18@nyu.edu
}

\begin{abstract}
Dynamic contrast-enhanced 4-D MR renography has the potential for broad clinical applications, but suffers from respiratory motion that limits analysis and interpretation. Since each examination yields at least over 10-20 serial 3-D images of the abdomen, manual registration is prohibitively laborintensive. Besides in-plane motion and translation, out-of-plane motion and rotation are observed in the image series. In this paper, a novel robust and automated technique for removing out-of-plane translation and rotation with sub-voxel accuracy in 4-D dynamic MR images is presented. The method was evaluated on simulated motion data derived directly from a clinical patient's data. The method was also tested on 24 clinical patient kidney data sets. Registration results were compared with a mutual information method, in which differences between manually co-registered time-intensity curves and tested time-intensity curves were compared. Evaluation results showed that our method agreed well with these ground truth data.
\end{abstract}

\section{Introduction}

Single kidney glomerular filtration rate and split renal function can be measured by gadolinium-enhanced MR renography. Despite the fact that the kidney is a 3-D organ, most previous animal and clinical studies have been restricted to serial 2-D MRI data [1]. With three-dimensional magnetic resonance renography [2, 3], 3-D MR acquisitions are recorded repeatedly for at least 4 minutes after intravenous injection of a low dose of gadopentetate dimeglumine. In this context, image analysis of perfusion images aims to construct representative time-intensity curves from specified regions of interest such as the renal cortex, medulla, and collecting system. When patients cannot hold their breath reproducibly during perfusion data acquisition, accurate computation of time-intensity curves becomes complicated because of image misalignment over time. In an earlier study [2], three-dimensional registration and segmentation of all images were performed separately for each kidney by two investigators. For each case, manual registration and segmentation required 
approximately 2-3 hours at the workstation. For clinical applications, this workload is prohibitively time- and labor-intensive. Therefore, automated and semi-automated image registration techniques to correct respiration motion are of great clinical interest. There has been little work related to the registration of dynamic renal perfusion MRI data in which registration in time series is restricted in 2-D plus time and in-plane motion only $[1,4,5]$. Automated full 3-D serial image registration remains an unsolved problem especially in the context of internal organs [6-9]. We propose a novel fully automated four-dimensional (3-D plus time) MRI renography registration framework based on wavelet and Fourier transforms (WTFT). First, a preprocessing of denoising is employed using edge-preserving anisotropic diffusion; secondly, an edge detection is implemented using a 3-D overcomplete dyadic wavelet expansion; thirdly, based on the previous edge images, a 3-D registration is applied using the Fourier transform; then an existing sub-voxel registration scheme, which was extended to $3-\mathrm{D}$, is used to refine the registration results. Our method was quantitatively evaluated by phantom studies as well as on 24 clinical data sets compared with manually registered ground truth. WTFT was also compared with an existing 3-D mutual information based registration method.

\section{Methodology}

\subsection{Anisotropic Diffusion}

If edge detection is applied directly on the original serial 3-D images, the edges caused by noise prevent the registration process from achieving accuracy. Therefore, we needed to apply a denoising process before edge detection. Here, we have applied a computationally efficient denoising filter based on anisotropic diffusion previous developed by Duan et al. [10].

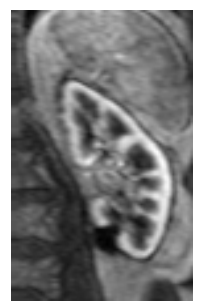

(a)

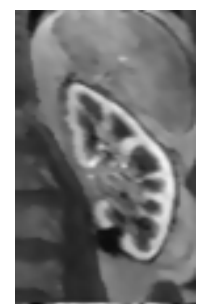

(b)

Fig. 1. The effect of anisotropic diffusion comparison (coronal view): (a) original image; (b) processed image

\subsection{Wavelet Edge Detection}

Due to the gadopentetate dimeglumine perfusion process, intensities of serial images change with time, therefore, it is unreliable to use intensity images directly. Instead, we can use edge information which is preserved fairly well in the serial 3-D image volumes. Compared with gradient and 3-D Sobel edge detection, wavelet transforms, which can also be used for edge detection, provide smooth and strong edge detection 
results. One way of implementing a multi-dimensional discrete dyadic transform is to use a filter bank scheme with separable wavelet bases [11]. Since the research in this paper focuses on three-dimensional processing, we used a three-dimensional discrete dyadic transform. We selected the modulus at level 2 for registration. A comparison of the three different edge detection methods is shown in Figure 2.

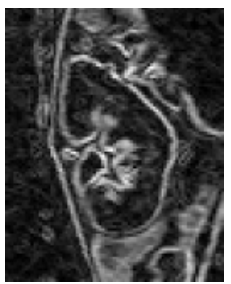

(a)

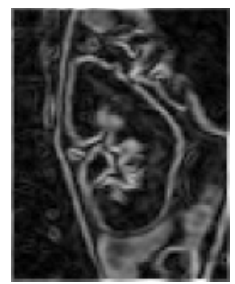

(b)

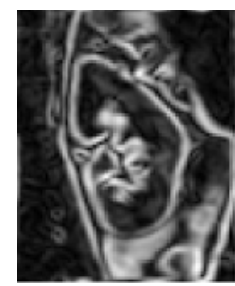

(c)

Fig. 2. A sample slice (coronal view) to compare different edge-detection methods: (a) gradient; (b) 3-D Sobel; (c) 3-D over-complete dyadic wavelet transform modulus

\subsection{Fourier Based Registration}

Using edge images acquired from previous step, a 4-D registration framework was accomplished by considering the first frame the reference as a 3-D object; the following 3-D frames were registered to the first one. Our work utilized a 3-D motion correction method based on the Fourier transform. The procedure can lead to an unsupervised 3-D rigid body registration method. One of the benefits of the method is that it makes use of all available information instead of limited features from the images. This makes the procedure very robust. Let $f(x, y, z)$ be a 3-D volume data, and let $g(x, y, z)$ be a translated and rotated version of $f(x, y, z)$, then

$$
g(x, y, z)=f(R x+t)
$$

where $t \in R^{3}$ is a translation vector, and $R \in S O(3)$ is a rotation matrix.

The three-dimensional Fourier transform is defined as:

$$
\mathfrak{F}[f(x, y, z)]=\int_{-\infty}^{\infty} \int_{-\infty}^{\infty} \int_{-\infty}^{\infty} f(x, y, z) e^{-j 2 \pi\left[w_{x} x+w_{y} y+w_{z} z\right]} d x d y d z .
$$

According to the property of the Fourier transform,

$$
\mathfrak{F}[g]\left(w_{x}, w_{y}, w_{z}\right)=R \mathfrak{F}[f]\left(w_{x}, w_{y}, w_{z}\right) e^{j 2 \pi\left[w_{x} x+w_{y} y+w_{z} z\right] R t}
$$

and

$$
\left|\mathfrak{F}[g]\left(w_{x}, w_{y}, w_{z}\right)\right|=\left|R \mathfrak{F}[f]\left(w_{x}, w_{y}, w_{z}\right)\right| .
$$

From above equations, we can see that the estimation of rotation has been decoupled from the estimation of translation. Thus the first estimation of $R$ should be implemented before the estimation of $t$. In three dimensional spaces, the rotation cannot be expressed in polar or spherical coordinates, in which case it would be reduced to a translation and be estimated by phase-correlation [8] as in the $2-\mathrm{D}$ case. In other words, whereas rotation in 2-D space can be completely expressed 
by one angle, in order to represent a rotation in 3-D space, three angles are needed (Euler's rotation theorem). Rodrigues' rotation formula is adopted, which gives an efficient method for computing the rotation matrix $R \in S O$ (3) ( $S O(3)=\left\{R \in \mathbb{R}^{3 \times 3}, R^{-1}=R^{T}, \operatorname{det}(R)=1\right\}$ is a group of the 3-D special orthogonal matrices) corresponding to a rotation by an angle $\psi \in \mathbf{R}$ about a rotation axis specified by the unit vector $\widetilde{\omega}=\left(\omega_{x}, \omega_{y}, \omega_{z}\right) \in \mathbb{R}^{3}$. Then $R$ is given by

$$
R=\left[\begin{array}{lll}
\cos \psi+\omega_{x}^{2}(1-\cos \psi) & \omega_{x} \omega_{y}(1-\cos \psi)-\omega_{z} \sin \psi & \omega_{y} \sin \psi+\omega_{x} \omega_{z}(1-\cos \psi) \\
\omega_{z} \sin \psi+\omega_{x} \omega_{y}(1-\cos \psi) & \cos \psi+\omega_{y}^{2}(1-\cos \psi) & -\omega_{x} \sin \psi+\omega_{y} \omega_{z}(1-\cos \psi) \\
-\omega_{y} \sin \psi+\omega_{x} \omega_{z}(1-\cos \psi) & \omega_{x} \sin \psi+\omega_{y} \omega_{z}(1-\cos \psi) & \cos \psi+\omega_{z}^{2}(1-\cos \psi)
\end{array}\right]
$$

Since any unit vector in 3-D space can be expressed by two angles $(\theta, \phi)$, the rotation axis unit vector can be calculated by three angles $(\theta, \phi, \psi)$ :

$$
\omega_{x}=\cos \theta \cos \phi, \quad \omega_{y}=\sin \theta \cos \phi, \quad \omega_{z}=\sin \phi .
$$

Since the kidney is a fairly symmetric object in 3-D, if we use the same method in Lucchese's work [7], which is mainly designed for binary images, the solution of the rotation axis is not unique because the intensity projection from different directions may be equal to the projection on the rotation axis. Furthermore, projection loses the information in the spatial domain, where the intensity profile along a fixed direction would provide extra information to find the rotation axis. Instead of three steps, in this context, only two steps were applied: rotation matrix, $R$, estimation and translation vector, $t$, estimation.

\section{Step1. Recovering the rotation matrix}

By using the relationship between the Fourier transform magnitudes in equation(4) and to avoid the effects of aliasing introduced by rotation to the energy minimization procedure, we use the energy term as

$$
E=\iiint\left(\mathfrak{F}[g]\left(w_{x}, w_{y}, w_{z}\right)-\mathfrak{F}[R f]\left(w_{x}, w_{y}, w_{z}\right)\right)^{2} d w_{x} d w_{y} d w_{z}
$$

and the optimum rotation axis and rotation angle can be recovered by

$$
(\hat{\theta}, \hat{\phi}, \hat{\psi})=\underset{\theta, \phi, \psi}{\arg \min } E
$$

The minimization problem in equation (8) can be efficiently solved by the QuasiNewton Method [12].

\section{Step2. Recover translation vector.}

After the rotation estimation, the rotational version of $f, R f$, is calculated. Thus, the translation vector can be easily recovered by a phase-correlation technique:

$$
\widehat{\operatorname{corr}}=\frac{\mathfrak{F}[g]\left(w_{x}, w_{y}, w_{z}\right) \mathfrak{F}^{*}[R f]\left(w_{x}, w_{y}, w_{z}\right)}{\left|\mathfrak{F}[g]\left(w_{x}, w_{y}, w_{z}\right) \| \mathfrak{F}[R f]\left(w_{x}, w_{y}, w_{z}\right)\right|}=e^{j\left[w_{x} w_{y} w_{z}\right] \cdot t}
$$

where * denotes complex conjugate and - denotes vector dot product. The inverse Fourier transform of the right-hand side of equation (9) is the Dirac impulse function. So the translation vector can be trivially found by finding the position of that impulse 
function. According to the rotation and translation estimation, an aligned image from $g(x, y, z)$ to $f(x, y, z)$ can be denoted as $\tilde{g}(x, y, z)$ and $\widetilde{f}(x, y, z)$, whose spectrum are $\widetilde{F}\left(w_{x}, w_{y}, w_{z}\right)$ and $\widetilde{G}\left(w_{x}, w_{y}, w_{z}\right)$.

\subsection{Subvoxel Refinement in Frequency Domain}

Based on the integer voxel translation estimation and correction, a subvoxel refining process can be used to make more accurate registration results. A 2-D subpixel registration method put forward by Stone et al. [9] was extended to a 3-D framework in this article for the first time. The method requires integer voxel accuracy because it can only correct subvoxel misalignment.

From the property of the Fourier transform,

$$
\widetilde{G}=\mathfrak{F}\left[f\left(x+x_{0}, y+y_{0}, z+z_{0}\right)\right]=\widetilde{F} e^{-j(2 \pi / N)\left(w_{x} x_{0}+w_{y} y_{0}+w_{z} z_{0}\right)}
$$

i.e.

$$
\widetilde{F} / \widetilde{G}=e^{j(2 \pi / N)\left(w_{x} x_{0}+w_{y} y_{0}+w_{z} z_{0}\right)} .
$$

In other words, the phase of $\widetilde{F} / \widetilde{G}$ should be a plane in the $\left(w_{x}, w_{y}, w_{z}\right)$ space. Therefore, the subvoxel registration problem can be converted into a 3-D plane fitting problem, which can be solved by least square fitting. For any voxel in the $\left(w_{x}, w_{y}, w_{z}\right)$ space, in theory, the following equation holds:

$$
\operatorname{phase}(\widetilde{F} / \widetilde{G})=(2 \pi / N)\left(w_{x} x_{0}+w_{y} y_{0}+w_{z} z_{0}\right) .
$$

Using matrix representation, the above equation is equivalent to

$$
\frac{2 \pi}{N}\left[\begin{array}{lll}
w_{x} & w_{y} & w_{z}
\end{array}\right]\left[\begin{array}{lll}
x_{0} & y_{0} & z_{0}
\end{array}\right]^{T}=[\operatorname{phase}(\widetilde{F} / \widetilde{G})] .
$$

Equation (13) can be solved by pseudo inverse methods or singular value decomposition for the subvoxel translation vector $\left[x_{0}, y_{0}, z_{0}\right]^{T}$.

In both Fourier registration and subvoxel refinement process, a 3-D window is applied, which is recognized for eliminating the spurious introduction of highfrequency spectral energy due to the boundary effects [9]. We tried Blackman, Hann, and Tukey windows ( $\mathrm{r}=0.5$, which is $\mathrm{r}$ is the ratio of taper to constant sections), and we found that Blackman window worked the best for this dynamic renal data. Although in some time frames, when contrast agent intake is maximum and kidney boundaries are not clear, inner structures and outside edges surround the kidney, such as part of liver edges, will help the registration process.

\section{Experiments and Results}

\subsection{Simulated Clinical Study}

Based on a manually registered 4-D MR renography data set, a simulated data set with dimension [77 974020 ] and voxel resolution $1.66 \mathrm{~mm} \times 1.66 \mathrm{~mm} \times 2.5 \mathrm{~mm}$ was generated by translating and rotating the kidney. Simulated motions included head to 
feet (HF) translation, left to right (LR) translation, anterior to posterior (AP) translation and rotation (Rot) with respect to three different axes, represented in terms of $(\theta, \phi, \psi)$, where $(\theta, \phi)$ defined the axis of rotation; $\psi$ the angle of the rotation along that axis (Table 1).

The estimated errors in translation and rotation are shown in Figure 3. Translation estimation errors were lower than 1.4 voxels in all the directions with mean value $0.53 \pm 0.47,0.51 \pm 0.46$, and $0.60 \pm 0.41$ in $\mathrm{x}, \mathrm{y}$, and $\mathrm{z}$ direction respectively; for rotation, except for one case, the errors in the two angles representing the rotation axis were less than 0.5 degrees, and the errors in rotation angle were less than 2.5 degrees with mean value $0.003 \pm 0.003,0.07 \pm 0.26$, and $1.14 \pm 0.72$ degrees in $(\theta, \phi, \psi)$.

Table 1. Simulated Motion for Each Time Frame (T)

\begin{tabular}{llllll}
\hline T & Motion & T & Motion & T & Motion \\
\hline 1 & Baseline & 8 & LR $(-1.66 \mathrm{~mm})+(90,0,5)$ & 15 & HF $(3.32 \mathrm{~mm})$ \\
2 & AP(-2.5mm)+(0,90,-9) & 9 & AP $(2.5 \mathrm{~mm})$ & 16 & AP $(5.0 \mathrm{~mm})$ \\
3 & LR(3.32mm)+(90,0,-2) & 10 & Baseline & 17 & Baseline \\
4 & HF(6.64mm) & 11 & HF $(1.66 \mathrm{~mm})$ & 18 & HF(-4.98mm) \\
5 & $(0,0,-4)$ & 12 & HF(-1.66mm) & 19 & Baseline \\
6 & HF(-6.64mm) & 13 & Baseline & 20 & LR $(-4.98 \mathrm{~mm})$ \\
7 & Baseline & 14 & LR(6.64mm) & & \\
\hline
\end{tabular}

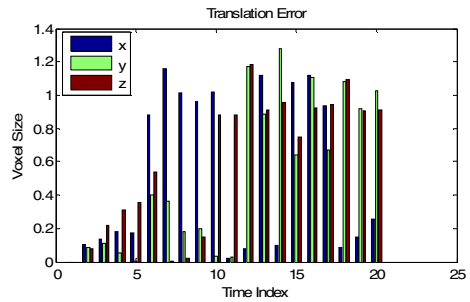

(a)

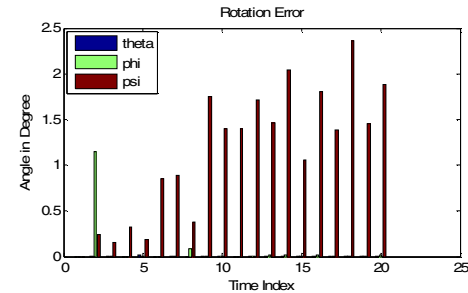

(b)

Fig. 3. Clinical phantom study results: (a) translation error; (b) rotation error

\subsection{Clinical Evaluation}

In order to evaluate the performance of our algorithm (WTFT) clinically, our algorithm was applied to 12 clinical patient datasets ( 24 kidneys in total), with manual registration and segmentation as ground truth. All datasets consisted of at least 41 3-D acquisitions, where each 3-D dataset comprised 40 interpolated partitions of $2.5 \mathrm{~mm}$ thickness, with inplane matrix of 256 and inplane voxel size $1.66 \times 1.66 \mathrm{~mm}$. After registration, the cortex, medulla, and collecting system were differentiated by applying manual segmentation labels on the first frame, assuming following frames were been correctly registered. The time-intensity curves of cortex, medulla, and collecting systems were calculated based on manual registration treated as ground 

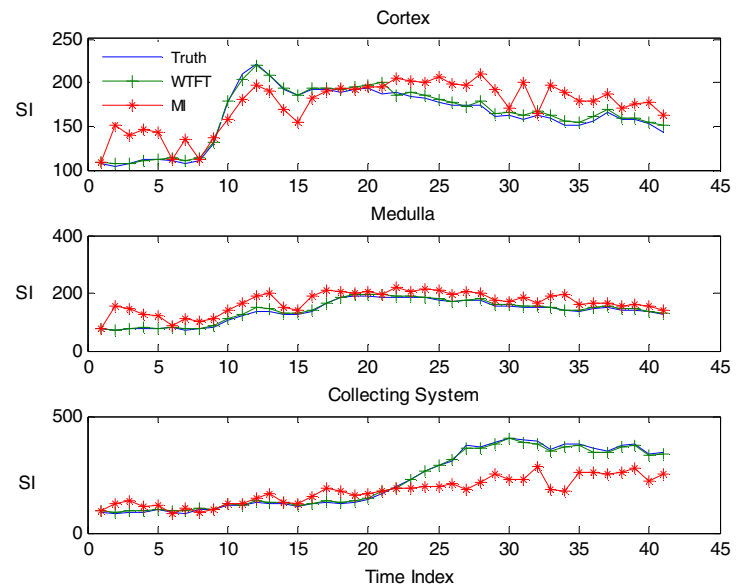

Fig. 4. Average intensity curves for one of the data sets using manual registration, WTFT, and mutual information registration

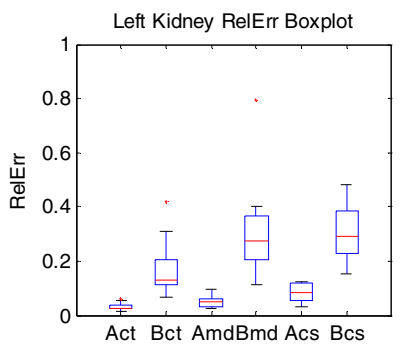

(a)

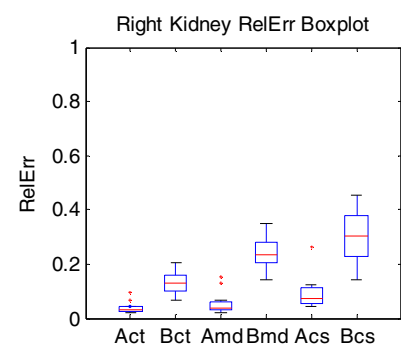

(b)

Fig. 5. Boxplot for RMS evaluation of the time-intensity curves generated from WTFT (A) and MI (B) methods. 'ct' stands for cortex; 'md' stands for medulla; 'cs'stands for collecting system. (a) left kidneys, (b) right kidneys.

Table 2. Significance values for Left and Right cortex, medulla and collecting systems for pairs of two methods

\begin{tabular}{|c|c|c|c|c|c|c|}
\hline \multirow{2}{*}{$\mathrm{P}$} & \multicolumn{3}{|c|}{ Left Kidney } & \multicolumn{3}{c|}{ Right Kidney } \\
\cline { 2 - 7 } & Cortex & Medulla & CollSys & Cortex & Medulla & CollSys \\
\hline WTFT/MI & $<0.0005$ & $<0.0005$ & $<0.0005$ & $<0.0005$ & $<0.0005$ & $<0.0005$ \\
\hline
\end{tabular}

truth and our automatic registration method. As a comparison, the time-intensity curves based on Viola-Wells Mutual Information (MI) $[13,14]$ were also calculated. In Figure 4, results from the three registration methods are shown for each kidney structure. Qualitatively, time-intensity curves based on WTFT registration are much closer to the ground truth than MI. To quantitatively evaluate the performance, root mean squared (RMS) relative errors of time-intensity curves between automatic registration methods, WTFT or MI, and ground truth were calculated and are shown 
in Figure 5. From the box-plot, the relative errors based on our WTFT method are much smaller than the MI methods in terms of average and standard deviation of RMS measurements. However, we note that both automated registration methods performed best for cortex but lower agreement for the collecting systems. The average errors for cortex, medulla and collecting systems in WTFT method were $3.24 \% \pm 1.41 \%, 5.31 \% \pm 2.19 \%$, and $8.23 \% \pm 3.35 \%$ respectively for the left and $3.99 \% \pm 2.23 \%, 5.67 \% \pm 4.13 \%$, and $9.26 \% \pm 5.94 \%$ for the right. Evaluation of the statistical differences of results from the two registration methods was performed at the significance level 0.05 with a Wilcoxon signed rank test for paired data. Significance values for the three tissue types for each pair of registration methods are reported in Table 2. Small p values (below 0.0005) indicate a significant statistical difference between the methods. From box plot, we can see WTFT had lower mean and smaller standard deviation compared with MI, so WTFT statistically performed better than MI.

\section{Conclusion}

In this paper, we proposed a novel fully automated four-dimensional MRI renography registration framework based on over complete dyadic wavelet and Fourier transform (WTFT), which was tested in terms of automation, robustness, and accuracy. Simulated motion studies and clinical evaluation studies were used to evaluate the new method. Comparison between different edge detection methods and comparison between WTFT and mutual information (MI) were performed to illustrate the effectiveness of the proposed scheme. An edge-preserving anisotropic diffusion operator was also introduced as a denoising method. Experimental results showed accurate registration results when compared to manual registration, by expert radiologists.

\section{References}

[1] Y. Sun, M.-P. Jolly, and J. M. F. Moura, "Integrated Registration of Dynamic Renal Perfusion MR Images," presented at IEEE International Symposium on Image Processing, ICIP'04, Singapore, 2004.

[2] V. S. Lee, H. Rusinek, M. E. Noz, P. Lee, M. Raghavan, and E. L. Kramer, "Dynamic Three-dimensional MR Renography for the Measurement of Single Kidney Function: Initial Experience," Radiology, vol. 227, pp. 289-294, 2003.

[3] J. Sajous, Y. Boykov, C. Chefdhotel, H. Rusinek, and V. Lee, "Performance of semiautomatic registration and segmentation on $4 \mathrm{D}$ contrast-enhanced magnetic resonance renography," presented at Radiological Society of North America 2003, 2003.

[4] G. Gerig, R. kikinis, W. Kuoni, G. K. v. Schulthess, and O. Kubler, "Semiautomated ROI Analysis in Dynamic MRI-Studies: PartI: Image Analysis Tools for Automatic Correction of Organ Displacement," IEEE Transaction on Image Processing, vol. 11, pp. 221-232, 1992.

[5] E. L. W. Giele, J. A. dePriester, J. A. Blom, J. A. d. Boer, J. M. S. v. Engelshoven, A. Hasman, and M. Geerlings, "Movement Correction of the Kidney in Dynamic MRI Scans Using FFT Phase Difference Movement Detection," Journal of Magnetic Resonance Imaging, vol. 14, pp. 741-749, 2001. 
[6] J. B. A. Maintz, "Retrospective Registration of Tomographic Brain Images." Utrecht, the Netherlands: Utrecht University, 1996, pp. 17.

[7] L. Lucchese, G. Doretto, and G. M. Cortelazzo, "A Frequency Domain Technique for Range Data Registration," IEEE Transaction on Pattern Analysis and Machine Intelligence, vol. 24, pp. 1468-1484, 2002.

[8] Y. Keller, A. Averbuch, and M. Israeli, "Pseudopolar-Based Estimation of Large Translations, Rotations, and Scalings in Images," IEEE Transaction on Image Processing, vol. 14, pp. 12-22, 2005.

[9] H. S. Stone, M. T. Orchard, E.-C. Chang, and S. A. Martucci, "A Fast Direct FourierBased Algorithm for Subpixel Registration of Images," IEEE Transaction on Geoscience and Remote Sensing, vol. 39, pp. 2235-2243, 2001.

[10] Q. Duan, E. D. Angelini, and A. Laine, "Assessment of visual quality and spatial accuracy of fast anisotropic diffusion and scan conversion algorithms for real-time threedimensional spherical ultrasound," presented at Proceedings of SPIE, Medical Imaging 2004: Ultrasonic Imaging and Signal Processing, 2004.

[11] Y. Jin, "Multi-Scale Processing of Tomographic Images Using Dyadic Wavelet Expansions," in Biomedical Engineering. New York: Columbia University, 2004, pp. 1221.

[12] D. F. Shanno, "Conditioning of Quasi-Newton Methods for Function Minimization," Matheamtics of Computing, vol. 24, pp. 647-656, 1970.

[13] ITK, www.itk.org.

[14] W. M. Well, P. Viola, and R. Kikinis, "Multimodal volume registration by maximzation of mutual information," presented at Proc. Med. Robot. Comp. Assist. Surg., Baltimore, 1995. 